\title{
Non-thermal particle effects on the $H \alpha$ and $H \beta$ line profiles in the 18 August 2002 solar flare
}

\author{
L. K. Kashapova ${ }^{1}$, P. Kotrč ${ }^{2}$, and Yu. A. Kupryakov ${ }^{2,3}$ \\ ${ }^{1}$ Institute of Solar-Terrestrial Physics, Irkutsk, Russia \\ ${ }^{2}$ Astronomical Institute, Ondřejov, Czech Republic \\ ${ }^{3}$ GAISH, MGU, Moscow, Russia
}

Received: 1 October 2007 - Revised: 7 May 2008 - Accepted: 7 May 2008 - Published: 15 October 2008

\begin{abstract}
We present results of the 18 August 2002 flare analysis as an example for developing a diagnostic tool for thermal and non-thermal processes in chromospheric lines. Taking into account the hard X-ray (HXR) emission, we attempted to derive the $\mathrm{H} \alpha$ and $\mathrm{H} \beta$ line properties which were caused by the non-thermal electron contribution and could be useful for diagnostic purposes. The flare itself was a sequence of harder and softer bursts in HXR and we investigated three flare kernels associated with them. Two of the kernels appeared simultaneously. This phase of the flare could be observed in a broad band of wavelengths (HXR, UV, optical and microwaves). Kernel 1 did not clearly coincide with any HXR source but its intensity increased with the HXR flux rise. The flare kernel 3 did not show any significant response in microwaves, however, the related HXR flux was comparable with the flux of the previous kernels. We carried out an analysis of the difference between the $\mathrm{H} \alpha / \mathrm{H} \beta$ profile rate in the line center at the distance of $0.5 \AA$ from the line center. Only kernel 2 showed parameter fluctuations that were related to HXR flux evolution. The supposition of the non-thermal electron effect on the $\mathrm{H} \alpha / \mathrm{H} \beta$ profile ratio was confirmed only at the kernel connected with the $25-50 \mathrm{keV}$ HXR source. We found further confirmation that the $\mathrm{H} \alpha / \mathrm{H} \beta$ line intensity ratio could be used as a diagnostic tool for nonthermal electron presence.
\end{abstract}

Keywords. Solar physics, astrophysics, and astronomy (Energetic particles; Flares and mass ejections) - Space plasma physics (Transport processes)

Correspondence to: L. K. Kashapova

(1kk@iszf.irk.ru)

\section{Introduction}

The energy storage, release and transport are still hot and enigmatic problems of solar physics. Nowadays, it is accepted that the solar flare energy is initially released in the corona and then transported by various means from the primary release site to the chromosphere. However, the role of denser chromospheric layers, where most of the flare energy is dissipated radiatively, is not minor but quite a substantial one (Heinzel, 2003; Hudson, 2007). Therefore, the understanding of the energy release/transport mechanisms in solar flares is closely connected with the lower atmosphere response. The accelerated or non-thermal particle beams are the fastest and most effective transportation agents. The presence of accelerated particles in solar flares is without any doubts. However, a definite mechanism of their effect on the solar atmosphere has not been accepted yet.

It is well known that the different parts of line profiles are formed in different layers of the solar atmosphere. Presence of non-thermal electron beams should result to a shift of the energy release maximum to deeper layers of the chromosphere, as compared to the thermal mechanism effect. In Kobylinskij and Zharkova (1996) calculations of radiative energy losses for the Balmer lines are given. A comparison of energy release distribution according to atmospheric density shows that in the case of a non-thermal electron beam the maximum energy release is shifted to layers with higher density. Nowadays, it is generally accepted that the density in solar atmosphere is not uniformly stratified; therefore, the position of the maximum energy release will depend on the atmosphere structure in a particular active region. The influence of this effect of non-thermal particle beams was investigated in Kašparová and Heinzel (2002), where the intensity ratios of Balmer series were calculated. The authors considered the intensity ratio at $0.4 \AA$ from the $\mathrm{H} \alpha$ line center and revealed a difference in cases of the thermal excitation

Published by Copernicus Publications on behalf of the European Geosciences Union. 


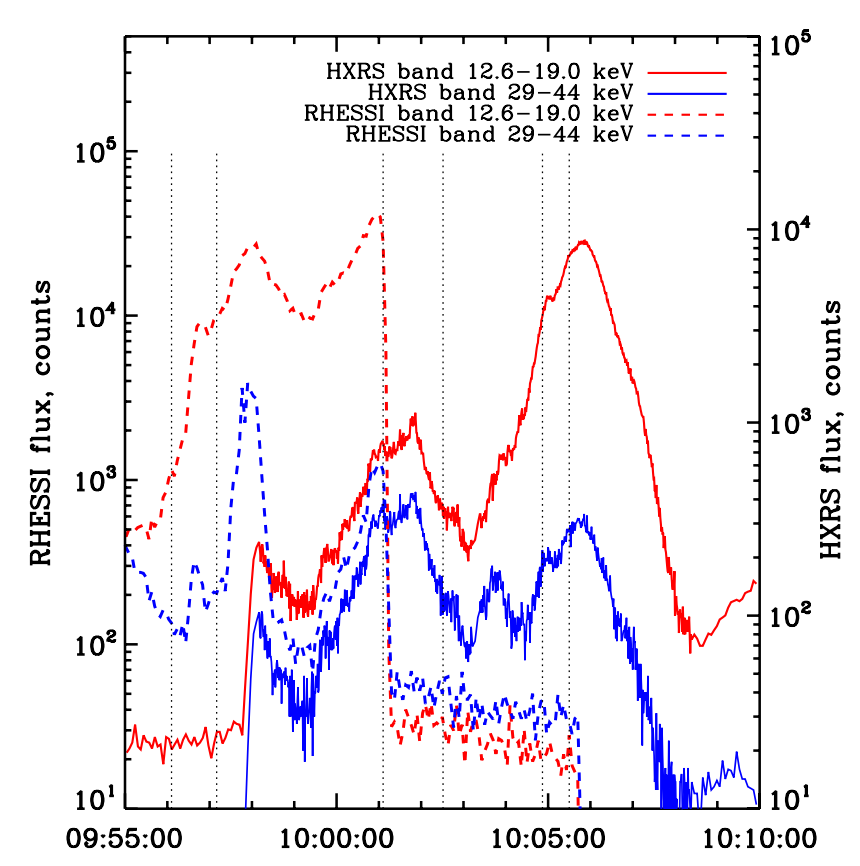

Fig. 1. Temporal evolution of the hard X-ray flux obtained by RHESSI and HXRS. The RHESSI data were transformed to HXRS energy bands. Edges of the three intervals covered by the MFS observations are marked by vertical dotted lines.

and of a non-thermal beam effect on ambient plasma. An injection of non-thermal electrons results in a depression of the ratio value in comparison with calculations without inclusion of the non-thermal collision rates. The deeper depression is caused by the beam with a harder power index and/or greater flux.

We analyzed the theoretical ratio profile used in Kašparová and Heinzel (2002) and found that the predicted effect is seen not only at $0.4 \AA$ but for the whole line ratio profile (Kashapova et al., 2005). Moreover, according to the calculated ratio profiles the maximum effect should be seen not at $0.4 \AA$ but at about $0.7 \AA$ from the line center. In Kashapova et al. (2005) the flare on 26 June 1999 was examined for the effect predicted in Kašparová and Heinzel (2002) and Kobylinskij and Zharkova (1996). The flare consisted of several kernels but only one of them coincided spatially with the HXR source (22.7-32.7 keV) and it definitely appeared as a result of the accelerated electron beam effect. In the kernel identified with the HXR source position we found that the profiles of the $\mathrm{H} \alpha / \mathrm{H} \beta$ line ratio displayed a peculiar pattern. Its shape was rather flat in the line center but with bumps in the wings. It was very similar to the theoretically predicted one (Kašparová and Heinzel, 2002) and it was called "sidelobes" (Kashapova et al., 2005). Certainly, the "sidelobes" effect could be caused not only by non-thermal electron beams. A plasma motion during the flare, a peculiar compact atmospheric density stratification and the other thermal-based reasons were able to produce the same line ratio profiles. Since we detected the "sidelobes" effect only in the kernel associated HXR source and thus with the accelerated particle injection, we attribute it as a consequence of non-thermal mechanisms. Positions of the "sidelobes" maxima were detected at $\pm 0.5 \AA$ from the line center and thus they differed slightly from the predicted ones. Such a disagreement could be caused by a difference between the atmospheric density distribution in the theoretical model used and in the particular active region. Therefore, the analysis of the ratio of Balmer lines based on the results obtained by Kobylinskij and Zharkova (1996), Kašparová and Heinzel (2002) and the first observational confirmation (Kashapova et al., 2005), could be a diagnostic tool for non-thermal electrons.

We chose the 18 August 2002 flare analysis as an example of thermal and non-thermal processes in chromospheric lines for several reasons. The aim of our study is to analyze properties of chromospheric lines which were influenced by the non-thermal electrons and could be useful for diagnostic purposes. The available flare observations embraced a wide range of electromagnetic spectrum, including the hard X-ray (HXR) and radio emission, which are very important for the non-thermal particle detection. The HXR flux energies were not higher than $100 \mathrm{keV}$ and this flare was softer than the flare studied earlier. But in this case we had the possibility to investigate several HXR peaks and try to reveal changes we were interested in.

The studied flare was a sequence of harder and softer bursts in HXR and we analyzed two of them. We also studied the chromospheric lines during the rising phase of the HXR burst with energy up to $100 \mathrm{keV}$ which was connected to a powerful $\mathrm{H} \alpha$ emission with extended chromospheric line wings. For analysis we used the parameters examined in a previous publication (Kashapova et al., 2007). When RHESSI interrupted observation, a subsequent HXR peak was registered by the Czech Hard X-Ray Spectrometer (HXRS). Thus, we took into account the possible influence of the two preceding harder HXR bursts and the effect of the subsequent HXR flux on the evolution of the chromospheric emission. We also tried to follow the possible scenario for the flare energy release/transport based on the UV and HXR observations and information on the magnetic field. The results are discussed both from their observational and theoretical aspects.

\section{Observations and data reduction}

The flare occurred on 18 August 2002, at 09:50-10:59 UT, (maximum at 10:05 UT) when the active region (AR) NOAA 10069 crossed the central meridian (S09, W13). According to the Solar Geophysical Data (SGD) it was of 1F importance in the $\mathrm{H} \alpha$-line and of M2.3 according to GOES. The X-ray observations of GOES-8 registered an emission from 


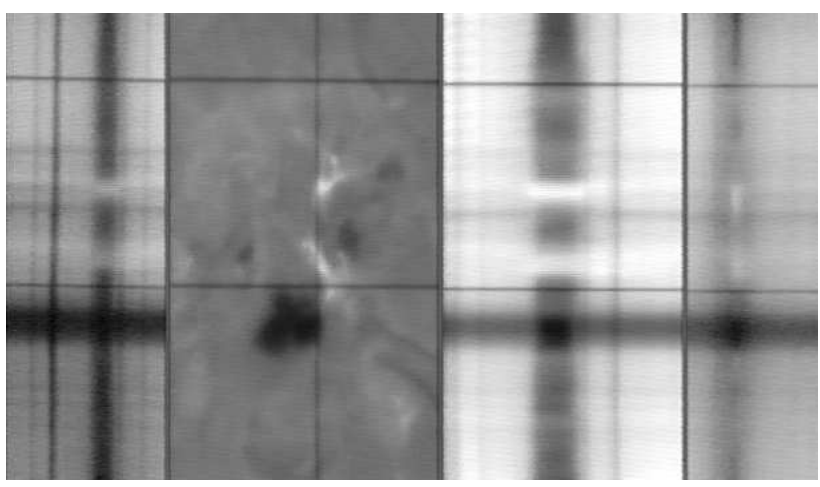

Fig. 2. The MFS observation of the flare kernels 1 and 2 taken at 09:56:30 UT when the first X-ray peak was observed. The image is composed of $\mathrm{H} \beta, \mathrm{H} \alpha$, and the infrared CaII spectrograms and $\mathrm{H} \alpha$ filtergram (the second section from the left, where North is up and West to the right). Notice that the $\mathrm{H} \alpha$ emission profiles were still rather narrow.

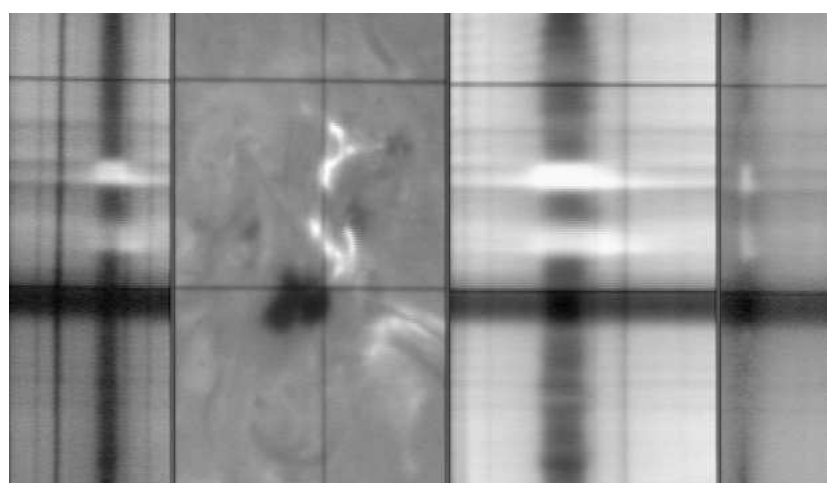

Fig. 3. The MFS composed observation of the two flare kernels at 10:01:24 UT - during the second rise of the X-ray. We notice quite a substantial broadening of the $\mathrm{H} \alpha$ emission profiles in the kernel.

09:54 UT to 10:09 UT with a maximum at 10:05 UT. We also had at our disposal the hard X-rays' data from RHESSI (Lin et al., 2002) and HXRS (Fárník et al., 2001), UV $195 \AA$ images obtained by TRACE (Handy et al., 1999), continuum and magnetic field images obtained by SOHO/MDI (Scherrer et al., 1995), and microwave flux from Radio Solar Telescope Network database (RSTN). RHESSI interrupted its observations at 10:01 UT by entering the Earth's shadow. Fortunately, practically at the right time (09:58 UT), the HXRS began observations. A combination of the data allows us to follow the HXR flux during the flare (Fig. 1) and to localize the HXR sources for the phase that started at about 09:59 UT.

We observed the optical flare spectra in $\mathrm{H} \alpha, \mathrm{H} \beta$ and the infrared CaII lines and also took simultaneous $\mathrm{H} \alpha$ slit-jaw images by the Ondřejov Multichannel Flare Spectrograph (MFS). This horizontal optical instrument enabled one to obtain simultaneous spectra in several lines with a theo-

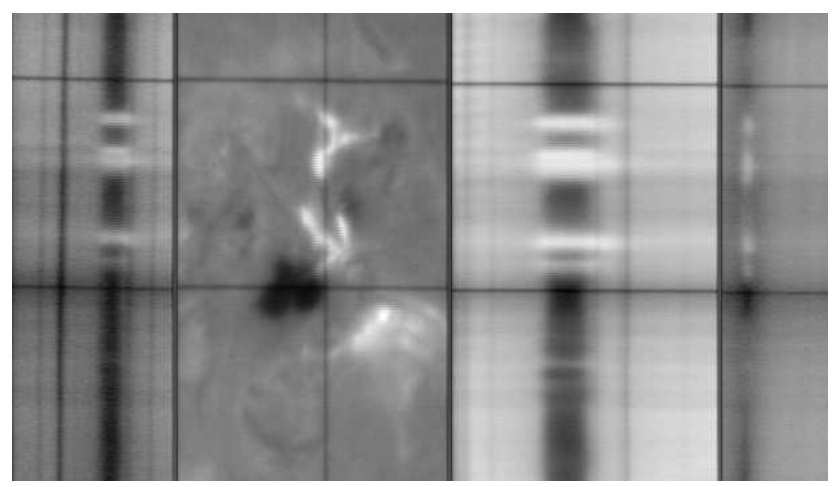

Fig. 4. The MFS composed observation of the two flare kernels at 10:04:19 UT - during the third rise of the X-ray. $\mathrm{H} \alpha$ emission profiles became a standard form again.

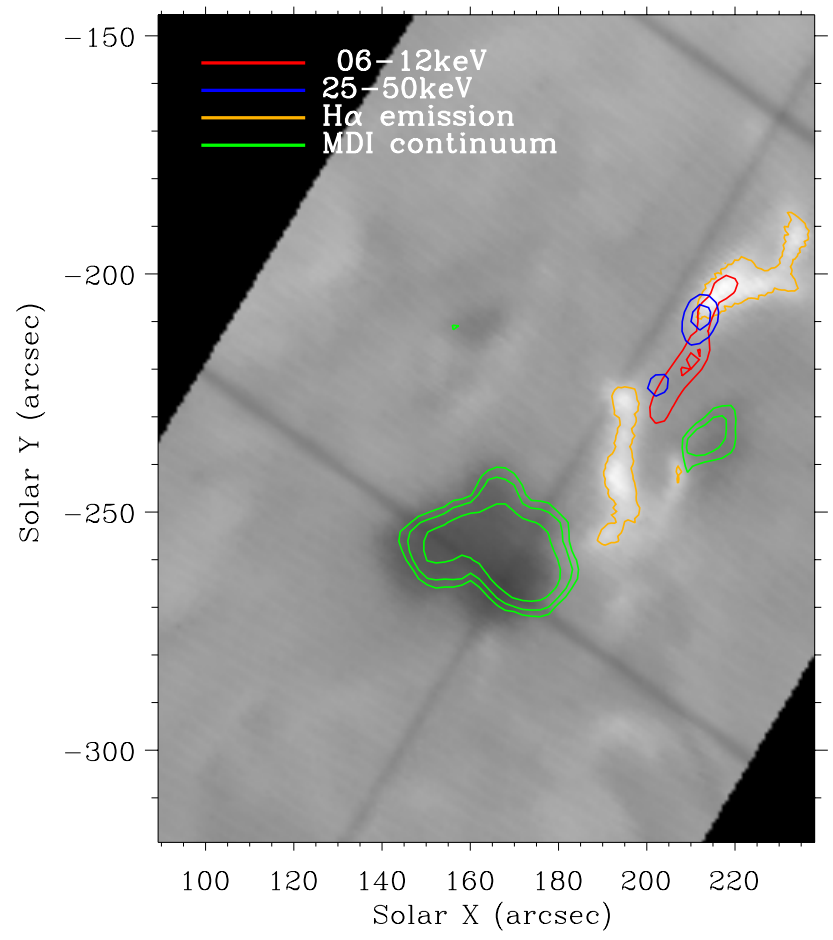

Fig. 5. The $\mathrm{H} \alpha$ slit-jaw image at $09: 57: 45$ UT overlaid by the MDI continuum spots and the HXR sources reconstructed in 09:56:3109:56:52 UT.

retical spatial resolution of 1 arcsec, but more practically, of $3 \mathrm{arcsec}$, due to observation degradation for the flare in question. The spectral resolution was about $40 \mathrm{~m} \AA$ in $\mathrm{H} \alpha$, while in $\mathrm{H} \beta$ it was about $30 \mathrm{~m} \AA$ and in the infrared CaII line it was $50 \mathrm{~m} \AA$. The temporal resolution was given by the video-camera detectors, i.e. 25 frames per second. The device was described by Valníček et al. (1959) and Kotrč (1997). According to observations in the HXR, the flare 


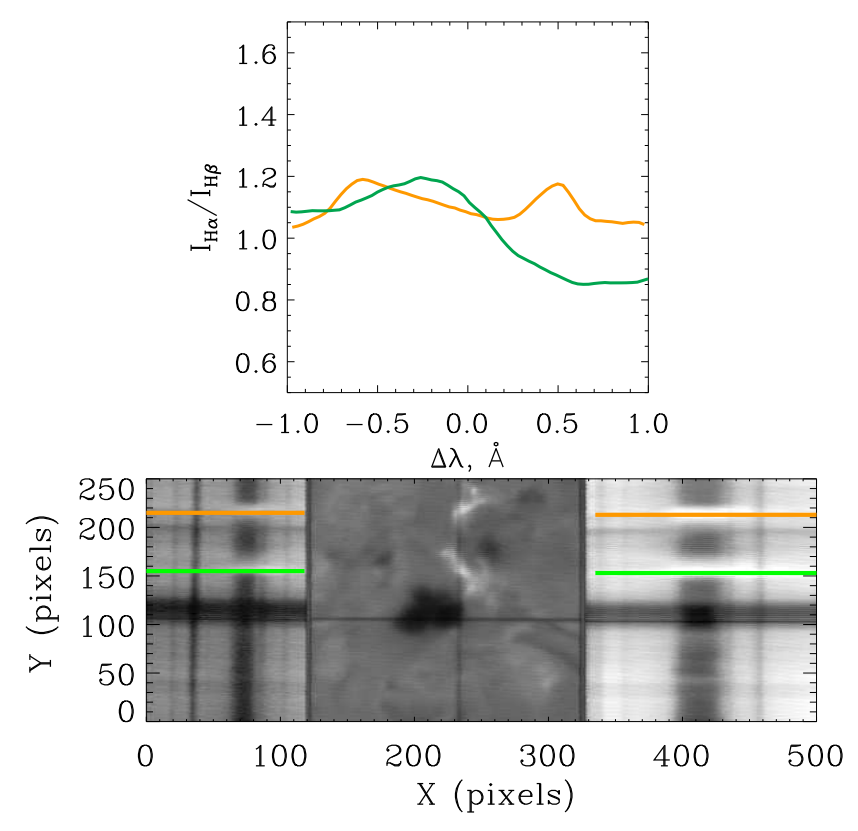

Fig. 6. Top: $\mathrm{H} \alpha / \mathrm{H} \beta$ ratio profiles at 09:56:45 UT for kernel 1 (green) and kernel 2 (orange). Bottom: MFS image composed of $\mathrm{H} \alpha$ spectrum, $\mathrm{H} \alpha$ slit-jaw and $\mathrm{H} \beta$ spectrum. The locations of kernels are marked by corresponding colors.

consisted of a sequence of events. Periods for which we have the continuous optical spectra observations are marked in the HXR temporal evolution chart in Fig. 1. The composed spectra and slit-jaw data registered on video cameras were digitized with a cadency 1 frame per $5 \mathrm{~s}$. The conclusions made by Heinzel (2003) and comparison of the observational data and results of the theoretical simulations in Zharkova et al. (2007) argued that such a temporal sampling would be quite satisfactory. Due to the detector properties the spectra were calibrated and treated individually to remove all important instrumental effects. For the reduction of the video-camera spectra concerning dispersion and intensity relations, the codes available at http://www.asu.cas.cz/ pkotrc/ SOFTWARE/software.html were used.

Typical optical spectra and $\mathrm{H} \alpha$ slit-jaw images for different moments of the flare can be seen in Figs. 2-4. They consist (from left to right) of the $\mathrm{H} \beta$ spectrum, $\mathrm{H} \alpha$ filtergram from the slit jaws, and $\mathrm{H} \alpha$ and the infrared CaII line spectra. All the image parts were obtained simultaneously. The infrared CaII line emission was too faint for a clear detection of any effect; therefore, we did not use it in the analysis. In order to fix the coordinate system of the studying region, we used SOHO/MDI intensitygrams and achieved the accuracy of the heliographic coordinates in about 2 arcseconds (see results in Fig. 5).

We are able to distinguish between three kernels. The first two kernels appeared in the period from 09:56 UT to 10:10 UT. As can be seen in Figs. 2-4, their shape hardly

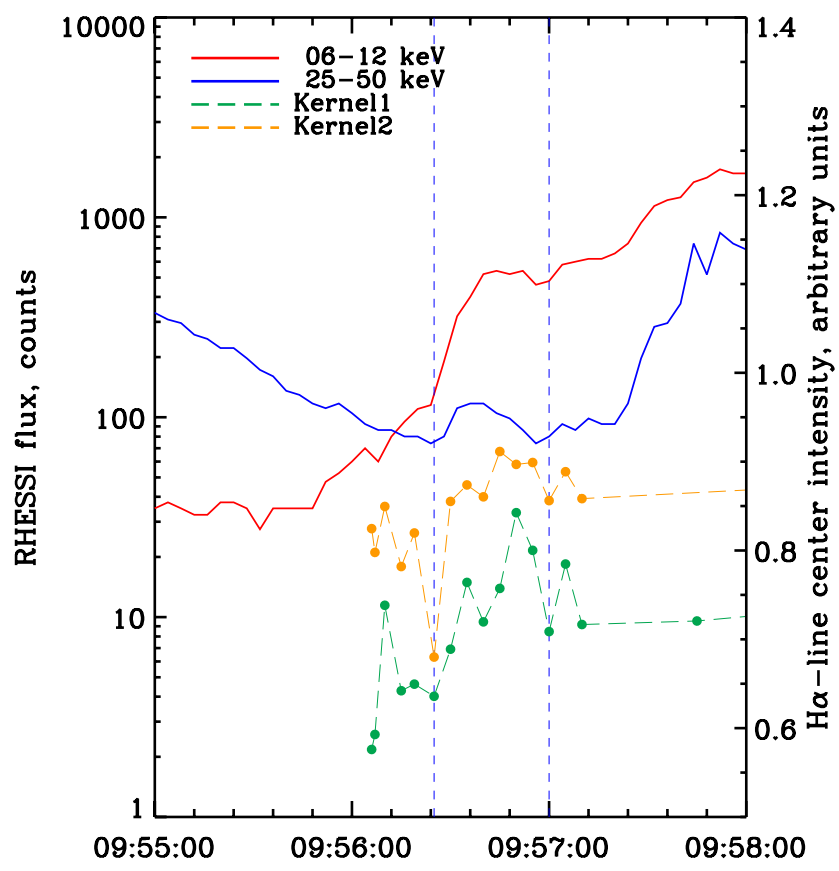

Fig. 7. Temporal evolution of hard X-ray flux from RHESSI and the $\mathrm{H} \alpha$-line center intensity of the kernels. The vertical dashed lines mark the beginning and end of the HXR burst.

changed during this phase of the flare. But the $\mathrm{H} \alpha / \mathrm{H} \beta$ ratio profiles showed different shapes (Fig. 6). Only kernel 2 (orange color), which was closely connected to the $25-50 \mathrm{keV}$ HXR source, showed the "sidelobes". Besides a comparison of the $\mathrm{H} \alpha$-line center emission evolution in the kernels with the HXR flux (Figs. 7 and 9), we tried to find a method to trace the "sidelobes" effect evolution. For this purpose the difference between the $\mathrm{H} \alpha / \mathrm{H} \beta$ rate in the line center and at the distance of $0.5 \AA$ from the line center were calculated. So the positive value of this parameter could mean that the rate profiles had a "thermal" form, the negative - a "non-thermal form" (Figs. 8 and 10).

The third kernel is shown at Fig. 3 (towards the southwest from the central spot). Its emission maximum coincided with the flare maximum (Fig. 4). The $\mathrm{H} \alpha$ image section was temporaly broadened in order to detect all the extremely wide line-wings. Therefore, the infrared CaII line observations (Fig. 12) were missing for the third kernel. Due to absence of RHESSI data for this phase, we were not able to localize the HXR sources and to analyze the HXR spectrum. We compared the optical data only with the HXR flux variations (Fig. 11).

\section{Discussion}

Two bright flare ribbons visible in $\mathrm{H} \alpha$ were visible in the first phase of the flare (Fig. 5). We investigated the evolution of 


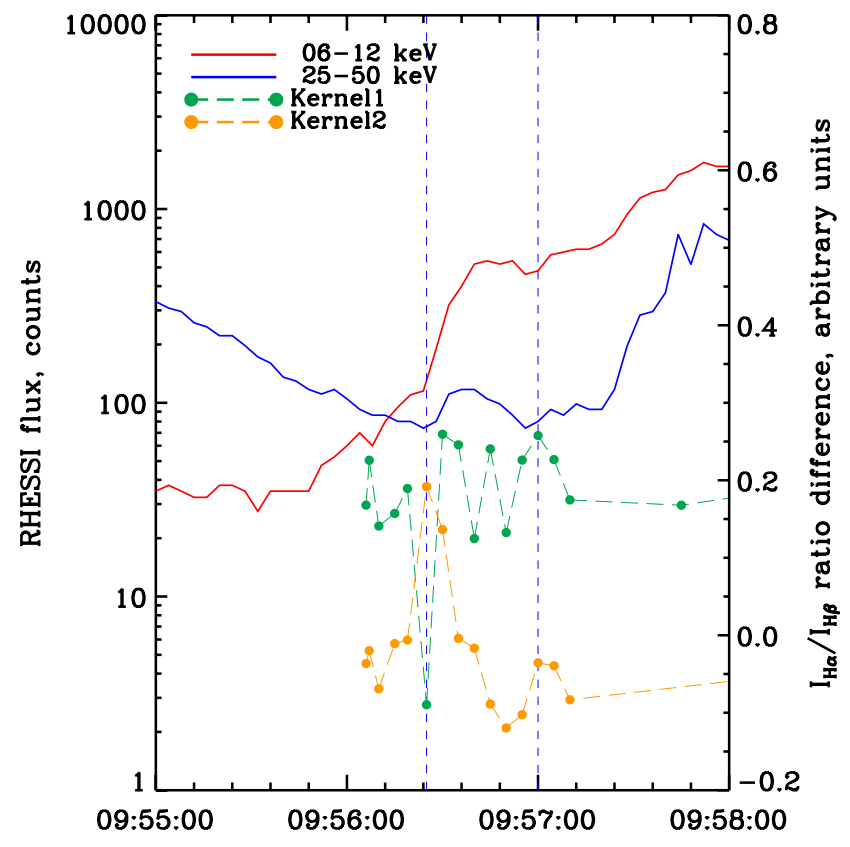

Fig. 8. Temporal evolution of HXR from RHESSI and the difference between the $\mathrm{H} \alpha / \mathrm{H} \beta$ ratio values in the line center and at the distance $0.5 \AA$ from the line centre. The vertical dashed lines mark the beginning and end of the HXR burst.

kernel 1 belonging to the southern ribbon (nearby the spot), and kernel 2 connected to the northern ribbon. The flare kernel 3 originated at 10:04 UT on the opposite side of the spot (Fig. 4 and the bottom panel on Fig. 12). The RHESSI data allowed us to reconstruct the HXR sources by the PIXON algorithm for the two first moments of observations and compare the HXR source locations with the location of kernels 1 and 2 and UV $195 \AA$ images obtained by TRACE (Fig. 13). We also carried out a study of HXR spectra for these two periods using the RHESSI software. The fitting by the thermal model agrees only with the observed HXR photon energy spectra up to $20 \mathrm{keV}$ for both periods. The power law energy spectra (connected with a velocity distribution of nonthermal electrons) defined well the HXR photon energy spectra between 20 and $100 \mathrm{keV}$, with the spectral index value of about 6 . The single power law also fitted well the HXR spectra from 3 to $100 \mathrm{keV}$ but with the spectral index equal to 6.6. We can carry out a more detailed analysis of the photon energy spectra but from the obtained results it is obvious that the non-thermal electrons were present in the flare.

Flare kernel 1 and kernel 2 (the bottom panel on Fig. 6) were observed in a broad region of wavelength from HXR and optical to microwaves. The $6-12 \mathrm{keV}$ HXR source at 09:56:47 UT looks like a composition of two loops whose footpoints coincided with the $25-50 \mathrm{keV}$ HXR sources (Fig. 13, left panel).The HXR sources became more compact in time and they were localized close to the kernel 2 .

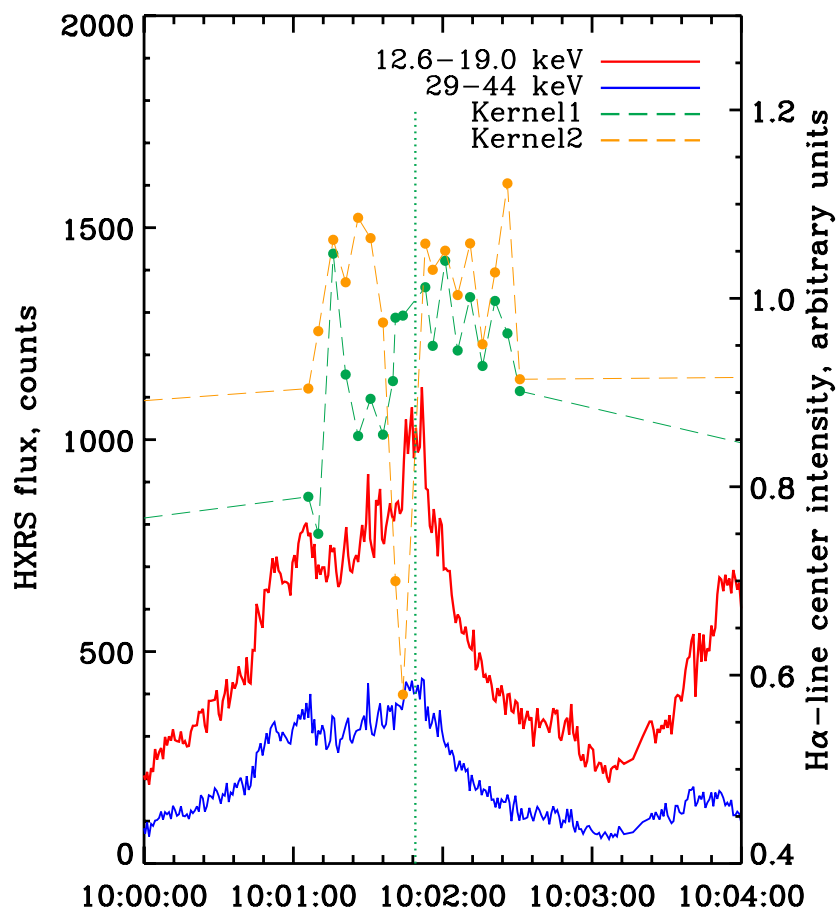

Fig. 9. Temporal evolution of HXR obtained by HXRS and the H $\alpha$ line center intensity of the kernels. The dash-dotted vertical green line shows the moment of missing MFS observations.

Concerning kernel 3, it did not show any significant response in microwaves while the related HXR flux was comparable with the flux of the previous kernels (Fig. 11). On the other hand, the emission in the optical spectra in kernel 3 was even more powerful (as can be seen in Fig. 3). It seems that the observed difference can be caused by the lower location of the initial energy-release site for kernel 3 (the density distribution was more compact) or a weak electron flux which gradually arrived from a remote energy release source connected to kernels 1 and 2. We would need a long loop to connect kernel 3 with the HXR sources. MDI magnetograms give us such a theoretical possibility but we do not see any loop like that at the available UV images.

As can be seen in Fig. 6, the ratio in kernel 2 shows a clear "sidelobes" shape. The rise of its intensity from 09:56 until 09:58 UT is in good agreement with the HXR flux increase and its location coincides with the HXR sources in $6-12 \mathrm{keV}$ and $25-50 \mathrm{keV}$. The other analyzed period of the kernel 2 evolution started at 10:01 UT. The $\mathrm{H} \alpha$-line center intensity also followed the HXR flux but did not demonstrate the same good agreement with the HXR flux evolution (Fig. 9). Kernel 1 did not coincide with any HXR source. While the 6-12 keV HXR source was located close to kernel 1 at 09:56 UT, the HXR source became more compact and coincided with kernel 2 only at 10:01 UT. 


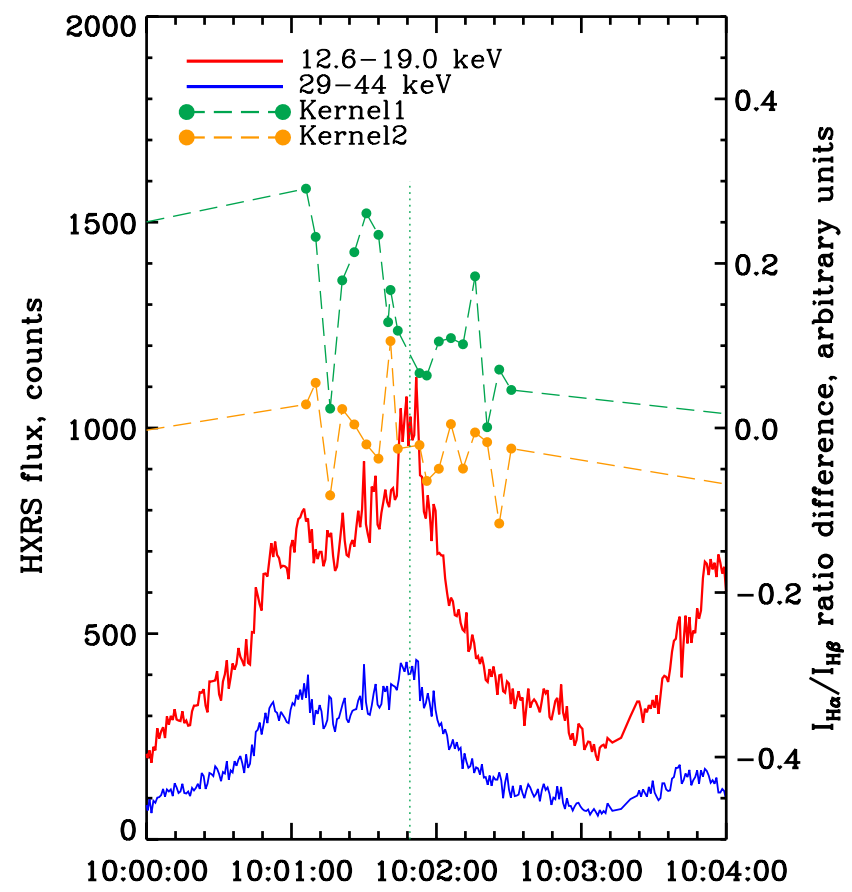

Fig. 10. Temporal evolution of HXR from HXRS and the difference between the $\mathrm{H} \alpha / \mathrm{H} \beta$ ratio values in the line center and at the distance $0.5 \AA$ from the line center. The dash-dotted vertical green line shows the moment of missing MFS observations.

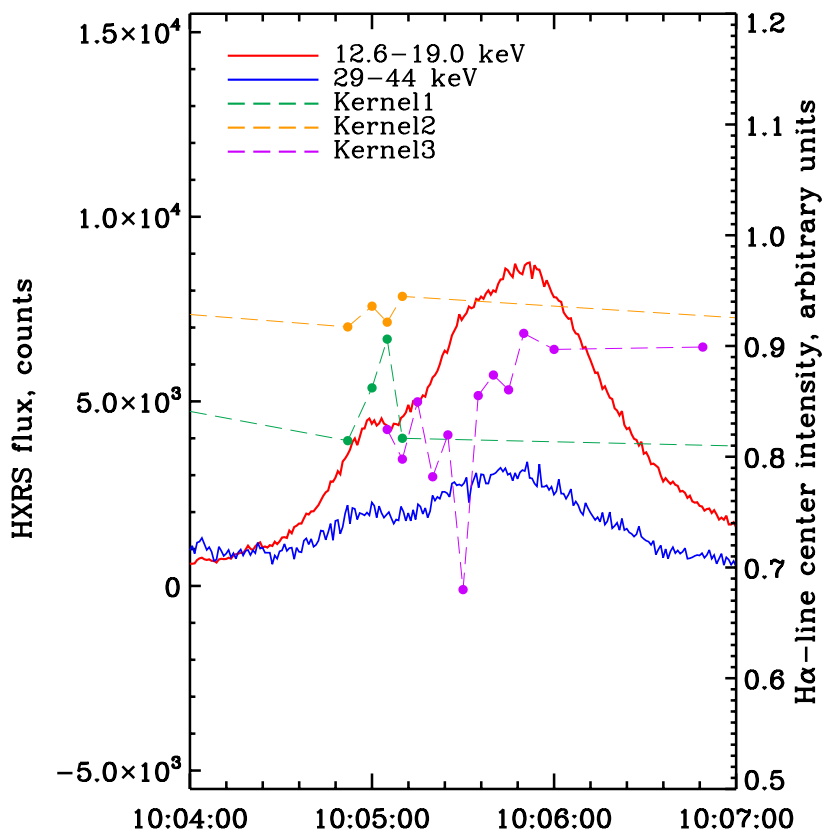

Fig. 11. Temporal evolution of HXR obtained by HXRS and H $\alpha$ line center intensity of the kernels.
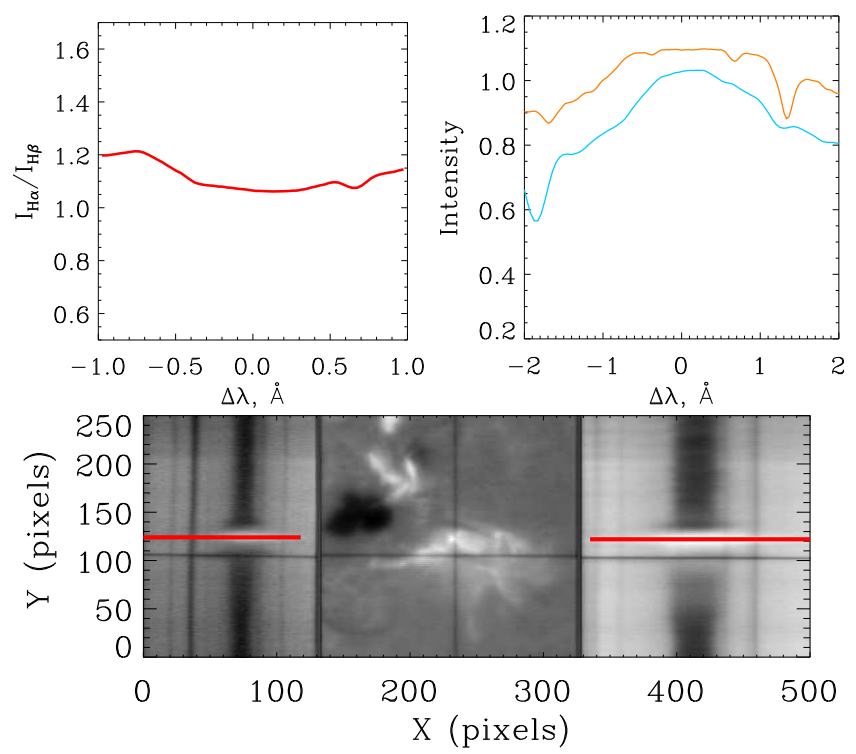

Fig. 12. Top: left $-\mathrm{H} \alpha / \mathrm{H} \beta$ ratio profiles for kernel 3 at 10:06:00 UT. Right - $\mathrm{H} \alpha$ (orange solid line) and $\mathrm{H} \beta$ (light greenblue solid line) profiles at the position marked at the bottom composed picture. Intensity of each line is related to the local continuum.

The behavior of the profile rate difference deserves a special attention. The difference in kernel 1 is mostly positive but the difference in kernel 2 changes from negative to positive values after the next HXR peak. We would like to point out some moments when the ratio difference in kernel 2 became positive. This could be a result of a gas-dynamic process that affects temporally the heating of the upper layers. All parameters corresponding to the non-thermal electron effects (both "classical" (Heinzel, 2003) and those suggested in Kashapova et al. (2007) were found in kernel 2.

Evolution visible in UV images overlayed by contours of $\mathrm{H} \alpha$ emission and HXR sources (shown on Fig. 13) confirms the possible existence of two loops. We would like to point out the small loop located within kernel 2, which is clearly distinguished in the Fig. 13 (right panel). It is associated with a footpoint marked by a $25-50 \mathrm{keV}$ HXR source in the left panel. Most possibly, the energy release source was located in this place. The second loop connected kernels 1 and 2. This loop is well fitted by a $6-12 \mathrm{keV}$ HXR source seen in the left panel (Fig. 13). On the right panel of Fig. 13, we can also see a third loop coming from kernel 2 but it wasn't connected with any HXR source. So, it is very probable that just the second loop interlinked kernel 1 with the energy release source and it was used for a electron flux transport. Possibly, the cutoff energy of the electron flux was up to $25 \mathrm{keV}$. Obviously, this energy is enough to excite emission in the $\mathrm{H} \alpha$-line center but not enough to penetrate into deep layers of the chromosphere. 

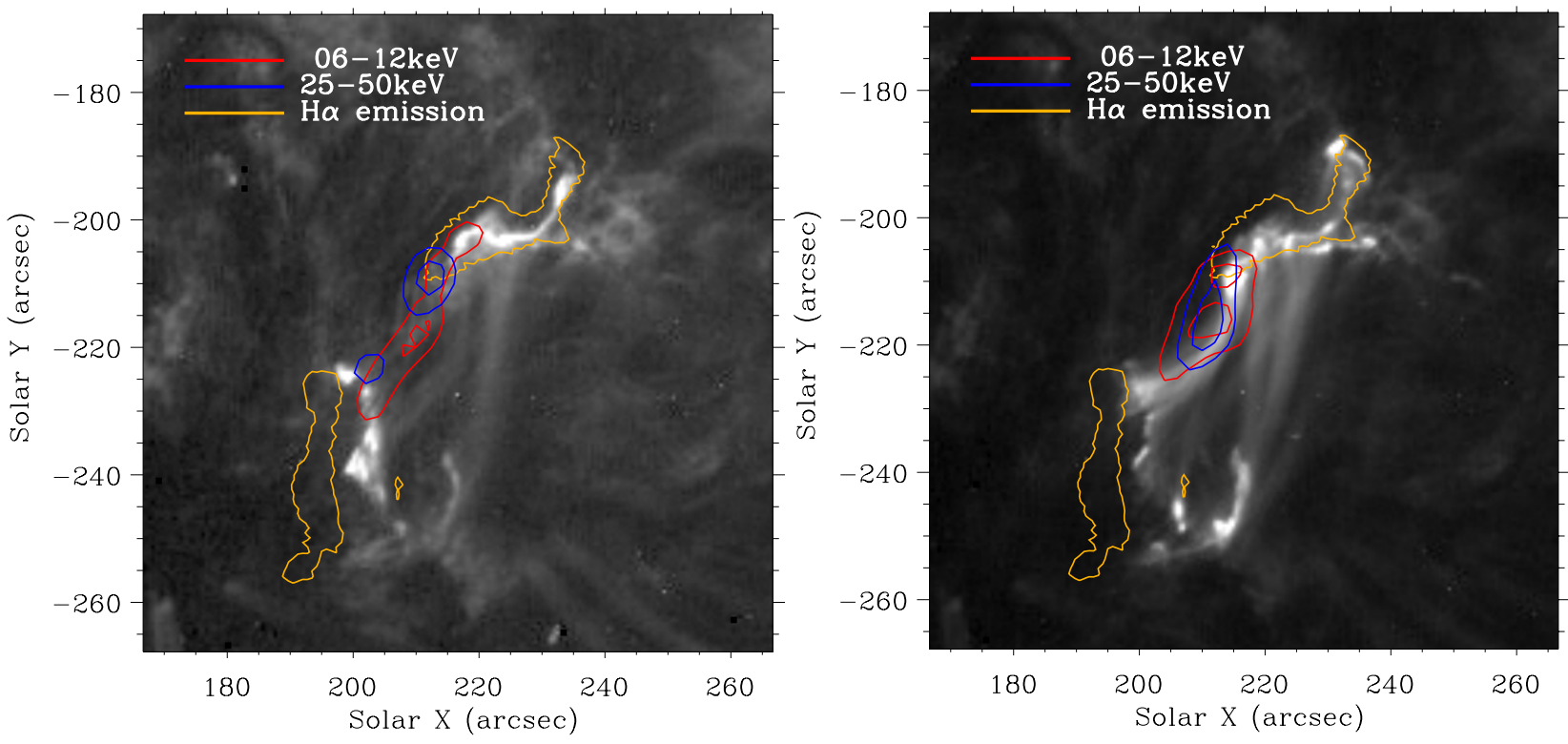

Fig. 13. UV $195 \AA$ by TRACE is overlaid by HXR sources and the $\mathrm{H} \alpha$ emission ribbons marked by orange contours at 09:56:47 UT (left panel) and 10:01:02 UT.

\section{Conclusions}

Based on the analysis carried out we can conclude:

- The analysis of the difference between the $\mathrm{H} \alpha / \mathrm{H} \beta$ profile rate in the line center and at the distance of $0.5 \AA$ from the line center revealed that kernel 1 and kernel 2 evolved in different ways. Kernel 1 stably demonstrated the ratio excess in the line center while kernel 2 showed fluctuations of this parameter.

- Hypotheses of non-thermal electron effect on the $\mathrm{H} \alpha / \mathrm{H} \beta$ profile ratio shape was confirmed by observation of kernels 2, in spite of the soft spectral index of the electron flux.

- Kernel 1 didn't clearly coincide with the HXR sources but its $\mathrm{H} \alpha$ intensity could follow the HXR growth.

- We found a further confirmation that the $\mathrm{H} \alpha / \mathrm{H} \beta$ line intensity ratio could be used as a diagnostic tool for nonthermal electron presence.

Acknowledgements. We are grateful to the SOHO/MDI and RHESSI teams who have provided a free access to their results. P. Kotrč appreciates support of the project AV0Z10030501 of the Astronomical Institute of the Academy of Sciences of the Czech Republic and the project No. 95 of the Czech-Slovak cooperation. The research by L. K. Kashapova was supported by the Russian Foundation of Basic Research (05-07-90209, 06-02-16239 and 0702-00101) and the Program of the Russian Academy of Sciences "Solar Activity and Physical Processes in the Sun-Earth System".

Topical Editor R. Forsyth and the authors thank two anonymous referees for their help in evaluating this paper.

\section{References}

Fárník, F., Garcia, H., and Karlický, M.: New Solar Broad-Band Hard X-Ray Spectrometer: First Results, Solar Phys., 201, $357-$ 372, 2001.

Handy, B. N., Acton, L. W., Kankelborg, C. C., et al.: The transition region and coronal explorer, Solar Phys., 187, 229-260, 1999.

Heinzel, P.: Understanding solar flares from optical observations: How do particle beams affect the lower atmosphere?, Adv. Space Res., 32, 2393-2402, 2003.

Hudson, H.: Chromospheric flares, ASP Conf. Series, 368, 365385, 2007

Kashapova, L. K., Kotrč, P., and Kupryakov, Yu. A.: Diagnostics of accelerated particle beams by spectral observations in Balmer series lines, ASP Conf. Series, 368, 431-436, 2007.

Kashapova., L. K., Kotrč, P., Kupryakov, Y. A., and Kašparová, J.: On an effect of particle beams on correlation between Balmer series lines in ESA SP-600 "The Dynamic Sun: Challenges for Theory and Observations", 2005.

Kašparová, J. and Heinzel, P.: Diagnostics of electron bombardment in solar flares from hydrogen Balmer lines, A\&A, 382, 688-698, 2002.

Kobylinskij, V. A. and Zharkova, V. V.: Hydrogen emission and radiative losses in the impulsive solar events, Adv. Space Res., 17, 110-114, 1996.

Kotrč, P.: Video Cameras in the Ondřejov Flare Spectrograph - Results and Prospects, Hvar Obs. Bull., 21, 97-108, 1997.

Lin, R. P., Dennis, B. R., Hurford, G. J., et al.: The Reuven Ramaty High-Energy Solar Spectroscopic Imager (RHESSI) Solar Phys., 210, 3-32, 2002.

Scherrer, P. H., Bogart, R. S., Bush, R. I., et al.: The Solar Oscillations Investigation - Michelson Doppler Imager, Solar Physics, 162, 129-188, 1995. 
Valníček, B., Letfus, V., Blaha, M., Švestka, Z., and Seidl, Z.: The flare spectrograph at Ondřejov, B. Astron. Inst. Czechosl., 10, $149,1959$.
Zharkova, V. V., Kashapova, L. K., Chornogor, S. N., and Andriyenko, O. V.: The multi-wavelength study of the effect of energetic particle beams on the chromospheric emission in the 25th July 2004 solar flare, J. Atmos. Sol. Terr. Phys., 39, 1483-1490, 2007. 